\title{
PARECERISTAS AD HOC 2021
}

O Comitê Editorial dos Cadernos CEDES agradece a colaboração dos pareceristas Ad hoc elencados a seguir, que emitiram pareceres e promoveram, com sua experiência e seu profissionalismo, a avaliação dos números temáticos publicados por este periódico no ano de 2021.

- Hanna Talita G. P. de Araújo Universidade Federal do Acre Rio Branco (AC), Brasil

- Yara Rondon Guasque Araújo Universidade Estadual de Santa Catarina Florianópolis (SC), Brasil

- Mônica C. Magalhães Kassar Universidade Federal de Mato Grosso do Sul

Campo Grande (MS), Brasil

- Ana Paula de Freitas

Universidade São Francisco

Bragança Paulista (SP), Brasil

- Lilian Nascimento

Universidade Estadual de Campinas

Campinas (SP), Brasil

- Marcia Mascia (USF)

Universidade São Francisco

Bragança Paulista (SP), Brasil

- Arlete Brito de Jesus

Universidade Estadual Paulista

Rio Claro (SP), Brasil

- Maria do Carmo Souza

Universidade Federal de São Carlos

- José Carlos Miguel

Universidade Estadual Paulista

Marília (SP), Brasil 